BULLETIN OF THE

AMERICAN MATHEMATICAL SOCIETY

Volume 79, Number 3, May 1973

\title{
THE GREEN FUNCTION OF A LINEAR DIFFERENTIAL EQUATION WITH A LATERAL CONDITION
}

\author{
BY CHAIM SAMUEL HÖNIG \\ Communicated by François Treves, November 13, 1972
}

Let $E$ be a Banach space. We consider systems of the form

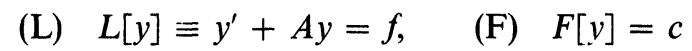

where $y \in \mathscr{C}^{(1)}([a, b], E), f \in \mathscr{C}([a, b], E), A \in \mathscr{C}([a, b], L(E)), F \in L[\mathscr{C}([a, b]$, $E), E]$ and $c \in E$. When the system has one and only one solution, for any $f \in \mathscr{C}([a, b], E)$ and $c \in E$, we show that it has a Green function, that is, a function $G:[a, b] \times[a, b] \rightarrow L\left(E, E^{\prime \prime}\right)$ such that $y \in \mathscr{C}^{(1)}([a, b], E)$ is the solution of $L[y]=f$ and $F[y]=0$ if and only if $y(t)=\int_{a}^{b} G(t, s) f(s) d s$. We exhibit the relations between $G, A$ and $F$. (F) is called a lateral condition; initial conditions and boundary conditions are particular instances of lateral conditions. The construction of $G$ uses a Riemann-Stieltjes integral representation for $F$, given in $\S 1$.

1. Analytic preliminaries. We consider always vector spaces over the complex field $\boldsymbol{C}$, but all results are valid for real vector spaces.

1. Given an interval $[a, b]$ of the real line, a division of $[a, b]$ is a finite sequence $d: t_{0}=a<t_{1}<\cdots<t_{n}=b$. We write $|d|=n$ and $\Delta d=$ $\sup \left\{\left|t_{i}-t_{i-1}\right||i=1,2, \ldots| d \mid,\right\} ; D$ denotes the set of all divisions of $[a, b]$.

2. Let $X, Y$ be Banach spaces; given $\alpha:[a, b] \rightarrow L(X, Y)$ and $d \in D$ we define

$$
S V_{d}[\alpha]=\sup \left\{\left\|\sum_{i=1}^{|d|}\left[\alpha\left(t_{i}\right)-\alpha\left(t_{i-1}\right)\right] x_{i}\right\| \mid x_{i} \in X,\left\|x_{i}\right\| \leqq 1\right\}
$$

and $S V[\alpha]=\sup \left\{S V_{d}[\alpha] \mid d \in D\right\}$.

We say that $\alpha$ is of bounded semivariation, and we write

$$
\alpha \in S V([a, b], L(X, Y)),
$$

if $S V[\alpha]<\infty$ (see for instance $[\mathbf{D}]$ and $[\mathbf{B}-\mathbf{K}])$.

Proposition 1. Given $\alpha \in S V([a, b], L(X, Y))$ and $f \in \mathscr{C}([a, b], X)$, there exists $F_{\alpha}[f]=\int_{a}^{b} d \alpha(t) \cdot f(t)=\lim _{\Delta d \rightarrow 0} \sum_{i=1}^{|d|}\left[\alpha\left(t_{i}\right)-\alpha\left(t_{i-1}\right)\right] \cdot f\left(\xi_{i}\right) \in Y$, where $\xi_{i} \in\left[t_{i-1}, t_{i}\right], i=1,2, \ldots,|d|$. We have $\left\|F_{\alpha}[f]\right\| \leqq S V[\alpha]\|f\|$ and hence

AMS (MOS) subject classifications (1970). Primary 34G05, 34A30, 34B05; Secondary $28 \mathrm{~A} 45,46 \mathrm{~A} 10$.

Key words and phrases. Linear differential equation, Green function, generalized boundary condition, lateral condition, bounded semivariation, vector valued Riemann-Stieltjes integral, representation of linear operators. 
$F_{\alpha} \in L[\mathscr{C}([a, b], X), Y]$ with $\left\|F_{\alpha}\right\| \leqq S V[\alpha]$.

ExAmple 1. If $Y=C$ then $S V([a, b], L(X, C))=B V\left([a, b], X^{\prime}\right)$ where $B V\left([a, b], X^{\prime}\right)$ is the space of functions $\alpha:[a, b] \rightarrow X^{\prime}$ that are of bounded variation, that is, such that $V[\alpha]=\sup \left\{V_{d}[\alpha] \mid d \in D\right\}<\infty$ where

$$
\begin{aligned}
V_{d}[\alpha] & =\sum_{i=1}^{|d|}\left\|\alpha\left(t_{i}\right)-\alpha\left(t_{i-1}\right)\right\| \\
& =\sup \left\{\left|\sum_{i=1}^{|d|}\left\langle x_{i}, \alpha\left(t_{i}\right)-\alpha\left(t_{i-1}\right)\right\rangle\right| \mid x_{i} \in X,\left\|x_{i}\right\| \leqq 1\right\} .
\end{aligned}
$$

By $B \widetilde{V}_{0}\left([a, b], X^{\prime}\right)$ we denote the space of all functions $\alpha \in B V\left([a, b], X^{\prime}\right)$ such that $\alpha(a)=0$ and $\alpha(t+)=\alpha(t)$ for $t \in] a, b[$. Endowed with the norm $V[\alpha], B \widetilde{V}_{0}\left([a, b], X^{\prime}\right)$ is a Banach space. We write

$$
B \widetilde{V}_{0}([a, b])=B \widetilde{V}_{0}([a, b], C) .
$$

In the usual way one proves the following

THEOREM 2 (RIESZ). $\mathscr{C}([a, b], X)^{\prime} \cong B \widetilde{V}_{0}\left([a, b], X^{\prime}\right)$; i.e., the mapping $\alpha \in B \widetilde{V}_{0}\left([a, b], X^{\prime}\right) \mapsto F_{\alpha} \in \mathscr{C}([a, b], X)^{\prime}$ is a linear isometry (i.e., $\left\|F_{\alpha}\right\|=$ $V[\alpha])$ of the first Banach space onto the second.

EXAmple 2. If $X=C$ we have $S V([a, b], L(C, Y))=B W([a, b], Y)$, where $B W([a, b], Y)$ is the space of functions $\alpha:[a, b] \rightarrow Y$ that are of weak bounded variation, that is, $W[\alpha]=\sup \left\{W_{d}[\alpha] \mid d \in D\right\}<\infty$ where

$$
W_{d}[\alpha]=\sup \left\{\left\|\sum_{i=1}^{|d|} \lambda_{i}\left[\alpha\left(t_{i}\right)-\alpha\left(t_{i-1}\right)\right]\right\|\left|\lambda_{i} \in C,\right| \lambda_{i} \mid \leqq 1\right\} .
$$

Definition. Let $Z$ be a Banach space;

$B \widetilde{W}_{0}\left([a, b], Z^{\prime}\right)=\left\{\alpha \in B W\left([a, b], Z^{\prime}\right) \mid z \circ \alpha \in B \widetilde{V}_{0}([a, b])\right.$ for every $\left.z \in Z\right\}$.

Endowed with the norm $W\left([\alpha], B \widetilde{W}_{0}\left([a, b], Z^{\prime}\right)\right.$ is a Banach space.

THEOREM 3. $L\left[\mathscr{C}([a, b]), Z^{\prime}\right] \cong B \widetilde{W}_{0}$

$$
\alpha \in B \widetilde{W}_{0}\left([a, b], Z^{\prime}\right) \mapsto F_{\alpha} \in L\left[\mathscr{C}([a, b]), Z^{\prime}\right]
$$

is a linear isometry (that is $\left\|F_{\alpha}\right\|=W[\alpha]$ ) of the first Banach space onto the second (for $\phi \in \mathscr{C}([a, b])$ we define $\left.F_{\alpha}[\phi]=\int_{a}^{b} \phi(t) d \alpha(t)\right)$.

3. Let $X$ and $Z$ be Banach spaces and $\alpha:[a, b] \rightarrow L\left(X, Z^{\prime}\right)$. Given $x \in X$ and $z \in Z$ we define $\alpha(x):[a, b] \rightarrow Z^{\prime}$ and $(z \circ \alpha)(x):[a, b] \rightarrow C$ by $\langle z, \alpha(x)(t)\rangle=\langle z, \alpha(t) x\rangle$ and $(z \circ \alpha)(x)(t)=\langle z, \alpha(t) x\rangle$.

We have

$$
B V\left([a, b], L\left(X, Z^{\prime}\right)\right) \subset S V\left([a, b], L\left(X, Z^{\prime}\right)\right) \subset B W\left([a, b], L\left(X, Z^{\prime}\right)\right)
$$


and we define

$$
\begin{aligned}
& S \widetilde{V}_{0}\left([a, b], L\left(X, Z^{\prime}\right)\right) \\
& \quad=\left\{\alpha \in S V\left([a, b], L\left(X, Z^{\prime}\right)\right) \mid(z \circ \alpha)(x) \in B \widetilde{V V}_{0}([a, b]) \text { for all } x \in X \text { and } z \in Z\right\} .
\end{aligned}
$$

Endowed with the norm $S V[\alpha], S \widetilde{V}_{0}\left([a, b], L\left(X, Z^{\prime}\right)\right)$ is a Banach space.

The following theorem is an extension of Riesz' representation theorems (Theorems 2 and 3):

Theorem 4. Let $X$ and $Z$ be Banach spaces. The mapping

$$
\alpha \in \widetilde{S V}_{0}\left([a, b], L\left(X, Z^{\prime}\right)\right) \mapsto F_{\alpha} \in L\left[\mathscr{C}([a, b], X), Z^{\prime}\right]
$$

is a linear isometry (i.e. $\left\|F_{\alpha}\right\|=S V[\alpha]$ ) from the first Banach space onto the second (see for instance [B-K, Satz 11]).

Corollary. Let $X$ and $Y$ be Banach spaces. For every

$$
F \in L[\mathscr{C}([a, b], X), Y]
$$

there is one and only one $\alpha \in \widetilde{S V}_{0}\left([a, b], L\left(X, Y^{\prime \prime}\right)\right)$ such that $F=F_{\alpha}$; we write $\alpha_{F}=\alpha$.

4. In what follows we extend the preceding results to locally convex topological vector spaces (LCTVS). We do not use these extended results in this paper.

Let $X$ and $Y$ be LCTVS; we denote by $P$ and $Q$ the set of all continuous seminorms defined on $X$ and $Y$, respectively. Given $\alpha:[a, b] \rightarrow L(X, Y)$, $p \in P, q \in Q$ and $d \in D$, we define

$$
S V_{q, p ; \alpha}[\alpha]=\sup \left\{q\left[\sum_{i=1}^{|d|}\left[\alpha\left(t_{i}\right)-\alpha\left(t_{i-1}\right)\right] \cdot x_{i}\right] \mid x_{i} \in X, p\left(x_{i}\right) \leqq 1\right\}
$$

and $S V_{q, p}[\alpha]=\sup \left\{S V_{q, p ; \alpha}[\alpha] \mid d \in D\right\}$. We write $\alpha \in S V_{q, p}([a, b], L(X, Y))$ if $S V_{q, p}[\alpha]<\infty$. We say that $\alpha$ is of bounded semivariation, and we write $\alpha \in S V([a, b], L(X, Y))$, if for every $q \in Q$ there is a $p \in P$ such that $S V_{q, p}[\alpha]<$ $\infty$; that is $S V([a, b], L(X, Y))=\bigcap_{q \in Q}\left[\bigcup_{p \in P} S V_{q, p}([a, b], L(X, Y))\right]$.

Proposition $1^{\prime}$. Let $X$ and $Y$ be LCTVS, $Y$ sequentially complete. Given $\alpha \in S V([a, b], L(X, Y))$ and $f \in \mathscr{C}([a, b], Y)$ there exists $F_{\alpha}[f]=$ $\int_{a}^{b} d \alpha(t) \cdot f(t)=\lim _{\Delta d \rightarrow 0} \sum_{i=1}^{|d|}\left[\alpha\left(t_{i}\right)-\alpha\left(t_{i-1}\right)\right] \cdot f\left(\xi_{i}\right) \in Y$, where $\xi_{i} \in\left[t_{i-1}, t_{i}\right]$. We have $F_{\alpha} \in L[\mathscr{C}([a, b], X), Y]$.

If $Y=C$ we get $S V([a, b], L(X, C))=B V\left([a, b], X^{\prime}\right)$ where $B V\left([a, b], X^{\prime}\right)$ is the space of functions $\alpha:[a, b] \rightarrow X^{\prime}$ that are of bounded variation, i.e., $B V\left([a, b], X^{\prime}\right)=\bigcup_{p \in P} B V_{p}\left([a, b], X^{\prime}\right) ; B V_{p}\left([a, b], X^{\prime}\right)$ denotes the space of functions $\alpha:[a, b] \rightarrow X^{\prime}$ such that $V_{p}[\alpha]<\infty$, where 


$$
V_{p}[\alpha]=\sup \left\{V_{p ; d}[\alpha] \mid d \in D\right\}
$$

and

$$
V_{p, d}[\alpha]=\sup \left\{\left|\sum_{i=1}^{|d|}\left\langle x_{i}, \alpha\left(t_{i}\right)-\alpha\left(t_{i-1}\right)\right\rangle\right| \mid x_{i} \in X, p\left(x_{i}\right) \leqq 1\right\} .
$$

By $B \widetilde{V}_{0}\left([a, b], X^{\prime}\right)$ we denote the space of all functions $\alpha \in B V\left([a, b], X^{\prime}\right)$ such that $x \circ \alpha \in B \widetilde{V}_{0}([a, b])$ for all $x \in X$.

Using the classical Riesz theorem (Theorem 2) we prove

THEOREM $2^{\prime} . \mathscr{C}([a, b], X)^{\prime} \cong \widetilde{B V}_{0}\left([a, b], X^{\prime}\right)$; i.e., the mapping

$$
\alpha \in \widetilde{B V}_{0}\left([a, b], X^{\prime}\right) \mapsto F_{\alpha} \in \mathscr{C}([a, b], X)^{\prime}
$$

is an isomorphism of the first vector space onto the second.

Let $X$ and $Z$ be LCTVS, $Z$ bornological; by $Z_{b}^{\prime}$ we denote its topological dual endowed with the strong topology. We define $S \widetilde{V}_{0}\left([a, b], L\left(X, Z_{b}^{\prime}\right)\right)=$ $\left\{\alpha \in S V\left([a, b], L\left(X, Z_{b}^{\prime}\right)\right) \mid z \circ \alpha \in B \widetilde{V}_{0}\left([a, b], X^{\prime}\right)\right.$ for all $\left.z \in Z\right\}$.

Using Theorem $2^{\prime}$ we prove

THEOREM $4^{\prime}$. Let $X$ and $Z$ be LCTVS, $Z$ bornological. The mapping

$$
\alpha \in S \widetilde{V}_{0}\left([a, b], L\left(X, Z_{b}^{\prime}\right)\right) \mapsto F_{\alpha} \in L\left[\mathscr{C}([a, b], X), Z_{b}^{\prime}\right]
$$

is an isomorphism of the first vector space onto the second.

Endowing the spaces above with their natural structure of LCTVS the algebraic isomorphisms in Theorems $2^{\prime}$ and $4^{\prime}$ become homeomorphisms.

2. The Green function. 1 . Given the differential operator $L$ defined in the introduction we denote by $R_{s}$ its resolvent, i.e., for every $s \in[a, b], R_{s}$ is the solution $R \in \mathscr{C}^{(1)}([a, b], L(E))$ of $d R / d t+A \circ R=0$ such that $R(s)=I_{E}$ (identical automorphism of $E$ ). We write $R(t, s)=R_{s}(t)$, where $t \in[a, b]$.

THEOREM 5. The solution of $L[y]=f, y(s)=c$ is given by $y(t)=$ $R(t, s) c+\int_{s}^{t} R(t, \sigma) f(\sigma) d \sigma$. (See, for instance, $[\mathbf{B}]$ or $[\mathbf{C}]$.)

2. Given $F \in L[\mathscr{C}([a, b], E), E]$ and $s \in[a, b]$, for every $x \in E$ we define $F\left[R_{s}\right] x=F\left[R_{s} x\right]$, hence $F\left[R_{s}\right] \in L(E)$. It is easy to show that $F\left[R_{s}\right]=$ $\int_{a}^{b} d \alpha(t) \circ R(t, s)$, where $\alpha=\alpha_{F}$. We write $J_{s}=J(s)=F\left[R_{s}\right]=F_{t}[R(t, s)]$.

The following theorem is easy to prove:

THEOREM 6. The following properties are equivalent:

(1) For every $f \in \mathscr{C}([a, b], E)$ and $c \in E$, the system $L[y]=f, F[y]=c$ has one and only one solution $y \in \mathscr{C}^{(1)}([a, b], E)$.

(2) For every $c \in E$ the system $L[y]=0, F[y]=c$ has one and only one solution $y \in \mathscr{C}^{(1)}([a, b], E)$. 
(3) The mapping $y \in\left\{u \in \mathscr{C}^{(1)}([a, b], E) \mid L[u]=0\right\} \mapsto F[y] \in E$ is an isomorphism of the first space onto the second.

(4) For every $s \in[a, b]$ we have $J_{s}=F\left[R_{s}\right] \in \operatorname{Aut}(E)$.

(5) There is an $s \in[a, b]$ such that $J_{s} \in \operatorname{Aut}(E)$.

From now on we suppose that the equivalent properties of Theorem 6 are verified.

It is immediate that

(1) For every $t, s \in[a, b]$ we have $R(t, s)=J(t)^{-1} \circ J(s)$.

(2) $F_{t}\left[J(t)^{-1}\right]=I_{E}$.

(3) $d J(t)^{-1} / d t=A(t) \circ J(t)^{-1}=0$.

Using (3) one can prove that

(4) For every $f \in \mathscr{C}([a, b], E)$ there exist the following integrals and we have

$$
\begin{aligned}
\int_{a}^{b} d \alpha(\tau) \circ J(\tau)^{-1}\left[\int_{a}^{\tau} J(s) f(s) d s\right] \\
=\int_{a}^{b}\left[\int_{s}^{b} d \alpha(\tau) \circ J(\tau)^{-1}\right] J(s) f(s) d s .
\end{aligned}
$$

THEOREM 7. If the properties of Theorem 6 are verified then

$$
y \in \mathscr{C}^{(1)}([a, b], E)
$$

is the solution of the system $L[y]=f, F[y]=c$ if and only if

$$
y(t)=J(t)^{-1} c+\int_{a}^{b} G(t, s) f(s) d s
$$

where

$$
G(t, s)=\hat{J}(t)^{-1} \circ\left[\int_{a}^{s} d \alpha_{F}(\tau) \circ J(\tau)^{-1}-Y(s-t) I_{E}\right] \circ J(s) .
$$

$\hat{J}(t)^{-1} \in L\left(E^{\prime \prime}\right)$ being the bitranspose of $J(t)^{-1} \in L(E)$ and $Y$ the Heaveside function. We have

(i) $G(t, s) \in L\left(E, E^{\prime \prime}\right)$;

(ii) $G(s+, s)-G(s-, s)=I_{E}$ for every $\left.s \in\right] a, b[$;

(iii) $G(t, b)=0 ; G(a, a)=-I_{E}$ and $G(t, a)=0$ for $a<t \leqq b$;

(iv) for every fixed $s \in[a, b], G$ is a continuous function of $t$, for $t \neq s$;

(v) for every fixed $t \in[a, b]$ and every $x \in E$, the function $s \in] a, b[\mapsto$ $G(t, s) \cdot x \in E_{\sigma\left(E^{\prime \prime}, E^{\prime}\right)}^{\prime \prime}$ is continuous on the right;

(vi) the function $G$ with these properties is unique.

SKETCH OF THE PROOF. If $y \in \mathscr{C}^{(1)}([a, b], E)$ is the solution of the system $L[y]=f, F[y]=c$ by Theorem 5 and (1) we have 


$$
\begin{aligned}
y(\tau) & =R(\tau, t) y(t)+\int_{t}^{\tau} R(\tau, s) f(s) d s \\
& =J(\tau)^{-1} J(t) y(t)+J(\tau)^{-1}\left[\int_{a}^{\tau} J(s) f(s) d s-\int_{a}^{t} J(s) f(s) d s\right] .
\end{aligned}
$$

Applying $F$ and using (2), the corollary of Theorem 4 and (4), one proves that

$$
c=J(t) y(t)-\int_{a}^{b}\left[\int_{a}^{s} d \alpha(\tau) \circ J(\tau)^{-1}\right] J(s) f(s) d s+\int_{t}^{b} J(s) f(s) d s,
$$

from which $(G)$ follows easily. Properties (i) to (v) follow from the expression for $G$ and the proof of (vi) uses Theorem 8 below.

3. Extensions of Theorem 7. Theorem 7 may be adapted to the case in which the system $L[y]=f, F[y]=0$ has one and only one solution for every $f \in \mathscr{C}([a, b], E)$. In this case $J_{t}^{-1}: E_{0}=F\left[\mathscr{C}^{(1)}([a, b], E)\right] \rightarrow E$ is continuous if and only if $E_{0}$ is a closed subspace of $E$.

THEOREM 8. The system $L[y]=f, F[y]=c$ where $f \in L_{1}([a, b], E)$ has one and only one solution $y \in L_{1}^{(1)}([a, b], E)$, given by $(\mathrm{G})$ (but now the integral is defined by continuous extension of $(\mathrm{G})$ from $\mathscr{C}([a, b], E)$ to $\left.L_{1}([a, b], E)\right)$.

THEOREM 9. If the system $L[y]=f, \hat{F}[y]=\hat{c}$, has one and only one solution $y \in \mathscr{C}^{(1)}([a, b], E)$ for every $f \in \mathscr{C}([a, b], E)$ and $\hat{c} \in E$, where $\hat{F} \in L\left[\mathscr{C}^{(1)}([a, b], E), E\right]$, then we can reduce it to a system $L[y]=f, F[y]=c$ that has also one and only one solution, where $F \in L[\mathscr{C}([a, b], E), E] . F$ and $c$ are given by

$$
F[y]=\hat{F}_{t}\left[y(a)-\int_{a}^{t} A(s) y(s) d s\right] \text { and } c=\hat{c}-\hat{F}_{t}\left[\int_{a}^{t} f(s) d s\right] .
$$

The preceding results may be extended to systems of the form

$$
L[y] \equiv A_{0}\left(A_{1} y\right)^{\prime}+B y=f, \quad F[y]=c
$$

where $A_{0}, A_{1}, B \in \mathscr{C}([a, b], L(E))$ and $A_{0}, A_{1}$ are invertible at every point $t \in[a, b]$. In this case $y \in D_{L}=\left\{u \in \mathscr{C}([a, b], E) \mid A_{1} u \in \mathscr{C}^{(1)}([a, b], E)\right\} ; D_{L}$ is endowed with the norm $\|u\|^{(L)}=\sup \left[\|u\|,\left\|\left(A_{1} u\right)^{\prime}\right\|\right]$ and $F \in L\left[D_{L}, E\right]$.

ADDED IN PROOF. The results of this paper may also be extended to half-open and open intervals, to the case where $A \in L_{1}^{\text {loc }}(] a, b[, L(E))$ and $F$ takes values in a Banach space different from $E$. The proofs will appear in $[\mathbf{H}]$.

\section{REFERENCES}

[B] N. Bourbaki, Éléments de mathématique. Part 1. Les structures fondamentales de 
l'analyse. Livre IV: Fonctions d'une variable réelle (Théorie élémentaire). Actualités Sci. Indust., no. 1132, Hermann, Paris, 1951. MR 13, 631.

[B-K] J. Batt und H. König, Darstellung linearer Transformationen durch vektorwertige Riemann-Stieltjes-Integrale, Arch. Math. 10 (1959), 273-287. MR 21 \# 7423.

[C] H. Cartan, Calcul différentiel, Hermann, Paris, 1967. MR 36 \# 6243.

[D] N. Dinculeanu, Vector measures, International Series of Monographs in Pure and Appl. Math., vol. 95, Pergamon Press, Oxford, 1967. MR 34 \#6011.

[H] C. S. Hönig, The abstract Riemann-Stieltjes integral and its applications to linear differential equations with generalized boundary conditions, Notas do Instituto de Matematica e Estatistica da Universidade de São Paulo, São Paulo, 1973.

Instituto de Matemàtica e Estatística, Universidade de São Paulo, São Paulo, BRAZIL 